\title{
Using International, Interprofessional Service Learning to Promote Transcultural Self-Efficacy and Interprofessional Attitudes in Health Science Students
}

\author{
Shana L. Cerny \\ University of South Dakota, Vermillion \\ Lana Svien \\ University of South Dakota, Vermillion \\ Julie Johnson \\ University of South Dakota, Vermillion \\ Bethany Hansmeier \\ University of South Dakota, Vermillion
}

Follow this and additional works at: https://encompass.eku.edu/jote

Part of the Occupational Therapy Commons, Pediatrics Commons, and the Physical Therapy Commons

\section{Recommended Citation}

Cerny, S. L., Svien, L., Johnson, J., \& Hansmeier, B. (2018). Using International, Interprofessional Service Learning to Promote Transcultural Self-Efficacy and Interprofessional Attitudes in Health Science Students. Journal of Occupational Therapy Education, 2 (1). https://doi.org/10.26681/jote.2018.020104 


\title{
Using International, Interprofessional Service Learning to Promote Transcultural Self-Efficacy and Interprofessional Attitudes in Health Science Students
}

\begin{abstract}
Interprofessional teams of occupational therapy, physical therapy, physician assistant, and dental hygiene students from the University of South Dakota participated in a service-learning experience in Guatemala. Student perceptions were measured using the Interprofessional Attitudes Scale and the Transcultural SelfEfficacy Tool. Qualitative data were obtained through written reflections and discussions during the immersion component of the service-learning experience. Students reported statistically significant improvements in transcultural self-efficacy as defined by their confidence in interviewing individuals from different cultures; their values, attitudes, and beliefs regarding cultural awareness, acceptance, appreciation, recognition, and advocacy; and their knowledge on how cultural factors influence care. Slightly different findings emerged concerning students' attitudes about interprofessional practice. Qualitative analysis of personal reflections showed that many students felt purposeful when working as part of an interprofessional team and appreciated the dialogue with other professionals when discussing patient care.
\end{abstract}

\section{Keywords}

Interprofessional practice, interprofessional education, Guatemala

\section{Creative Commons License}

\section{(a) $\oplus \Theta \Theta$}

This work is licensed under a Creative Commons Attribution-Noncommercial-No Derivative Works 4.0 License.

\section{Acknowledgements}

The authors would like to acknowledge Melissa Castillo, PA-C, Assistant Professor at the University of South Dakota, Vermillion; and Becca Fischer, OTD, OTR/L and Nicole Green, OTD, OTR/L, who were students at the University of South Dakota, Vermillion during the study. 


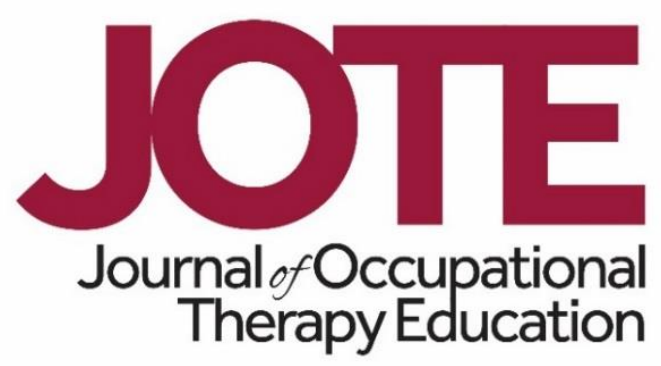

Volume 2, Issue 1

\title{
Using International, Interprofessional Service Learning to Promote Transcultural Self-Efficacy and Interprofessional Attitudes in Health Science Students
}

\author{
Shana Cerny, OTD, OTR/L, BCP, Lana Svien, PhD, PT, \\ Julie Johnson, MD, and Bethany Hansmeier, OT, OTR/L \\ University of South Dakota \\ United States
}

\begin{abstract}
Interprofessional teams of occupational therapy, physical therapy, physician assistant, and dental hygiene students from the University of South Dakota participated in a service-learning experience in Guatemala. Student perceptions were measured using the Interprofessional Attitudes Scale and the Transcultural Self-Efficacy Tool. Qualitative data were obtained through written reflections and discussions during the immersion component of the service-learning experience. Students reported statistically significant improvements in transcultural self-efficacy as defined by their confidence in interviewing individuals from different cultures; their values, attitudes, and beliefs regarding cultural awareness, acceptance, appreciation, recognition, and advocacy; and their knowledge on how cultural factors influence care. Slightly different findings emerged concerning students' attitudes about interprofessional practice. Qualitative analysis of personal reflections showed that many students felt purposeful when working as part of an interprofessional team and appreciated the dialogue with other professionals when discussing patient care.
\end{abstract}

\section{INTRODUCTION}

According to the 2010 census report, no single ethnic group will make up a majority of the United States' population by the year 2043. Minority populations are expected to more than double (116.2 to 241.3 million) during this period (United States Census Bureau, 2012). With this rise in diversity comes an increased need for culturallycompetent healthcare professionals who can provide quality care to diverse populations. Cultural competence refers to an individual's knowledge, skills, and attitudes regarding individuals from diverse backgrounds. However, many professionals believe cultural competence goes beyond knowledge and skill. Cultural competence 
should be thought of as "the continuous refinement and fostering of a type of thinking and knowing- a critical consciousness - of self, others, and the world" (Kumagai \& Lypson, 2009, p. 783).

Most allied health educational programs have educational standards in place to ensure some degree of cultural competence in their graduates. For example, several educational standards developed by the Accreditation Council for Occupational Therapy Education (ACOTE, 2012) include cultural criteria. Occupational therapy programs must teach students to consider factors that might bias assessment results, such as culture, disability status, and situational variables related to the individual and context (ACOTE Standard B.4.7). Culture must also be considered when providing population-based health interventions (ACOTE Standard B.3.5.). Likewise, the Liaison Committee on Medical Education (LCME, 2017), which sets the standards for medical student education, includes a standard regarding cultural competence and health care disparities; specifically, "The faculty of a medical school ensure that the medical curriculum provides opportunities for medical students to learn to recognize and appropriately address gender and cultural biases in themselves, in others, and in the health care delivery process" (LCME Standard 7.6). Physician assistant (PA) training programs have cultural competency standards that are required from their accrediting body, the Accreditation Review Committee for the Physician Assistant (ARC-PA, 2016), which states "the curriculum must include instruction to prepare students to provide medical care to patients from diverse populations" (ARC-PA Standard B1.06.). Some research indicates that multicultural educational practices are being utilized more frequently in health science education, but many programs report continued challenges (Brown, Munoz, \& Powell, 2011). While educational standards may help students understand the impact of diversity and culture on practice, they may not be enough to develop health care professionals who have good self-efficacy about their cultural competence.

International service learning may be one way to improve allied health students' cultural self-efficacy. International service learning occurs when students travel to different countries and cultures to provide services through partnerships with local organizations (Grusky, 2000). Service learning has a dual purpose; to address community needs in the environment the learning is taking place, and to meet the student's learning objectives through structured reflection (Jacoby, 2015). The two fundamental concepts of service learning are reflection and reciprocity. Service learning is based on the assumption that learning does not necessarily occur because of experiences, but rather personal reflection on those experiences. Reflection promotes a deeper understanding of the needs or issues being addressed and should encourage students to integrate their experiences and observations with existing knowledge. Reciprocity symbolizes the partnership between students, faculty, and the host community or organization. Service 
learning should balance student learning and community outcomes; mutually benefiting both parties (Jacoby, 2015).

Research shows that international service-learning experiences can increase cultural awareness (Humbert, Burket, Deveney, \& Kennedy, 2012; Kohlbry, 2016; Lawson \& Olson, 2017), confidence working with individuals from a different culture (Lawson \& Olson, 2012), and the cultural knowledge and skills of healthcare students (Kohlbry, 2016). Additionally, a study completed by Amerson (2010) found that international service learning experiences resulted in higher perceived cultural competence of students when compared to domestic projects. However, international service-learning programs can have negative implications as well. Grusky (2000) stated:

"Without thoughtful preparation, orientation, program development, and encouragement of study, and critical analysis and reflection, the programs can easily become small theaters that recreate historic cultural misunderstanding and simplistic stereotypes and replay, on a more intimate scale, the huge disparities in income and opportunity that characterize North-South relations today" (p. 858).

Noted implications include reinforcement of stereotypes, disruption of community relations, lack of sustainability, and cultural misunderstandings (Crabtree, 2013; Grusky, 2000; Lattanzi \& Pechak, 2011).

Each year, interprofessional teams of occupational therapy (OT), physical therapy (PT), PA, and dental hygiene students from the University of South Dakota participate in a service-learning experience in Guatemala. More than half of the Guatemalan population lives in poverty, with $13 \%$ living in extreme poverty (Central Intelligence Agency, 2016). A 36-year civil war, ending in 1996, left the Guatemalan health care system with limited financial and human resources (Commission for Historical Clarification, 1999) as indicated by having only 12.5 healthcare workers and 1.8 dentists per 10,000 individuals (United States Agency of International Development, 2015). Guatemala has a long history of discrimination and exploitation of the indigenous Mayan population with disparities existing between urban, Latino populations, and rural, Mayan populations (Commission for Historical Clarification, 1999). For example, there are approximately 25.7 healthcare workers per 10,000 individuals in urban areas and only 2.96 in rural areas (MSPAS, PAHO, WHO, 2013). Alternatively, Guatemala is a country rich in culture and history, allowing students to appreciate its traditional architecture, experience the rituals of both Mayan and Catholic cultures, and explore the natural beauty of pine forests and active volcanoes.

The phenomenon of interest in this study was the perceptions of health science graduate students regarding the impact of an international, interprofessional servicelearning trip on promoting cultural self-efficacy and attitudes toward interprofessional collaboration. We were particularly interested in how such an experience may have 
influenced the growth of healthcare students so they would become both academically trained and culturally sensitive professionals. Some of the indicators of the phenomenon were written reflections of the research participants indicating their perceptions about the impact of their daily experiences on their heart, their skills as a professional, and the purposefulness of their service to others. It was hypothesized that there would be a direct benefit to the students who participated on this trip, specifically, increased cultural awareness and improved attitudes for interprofessional practice.

\section{METHODS}

\section{Design}

This mixed methods study utilized a pretest/posttest and naturalistic type phenomenological design. The utilization of qualitative and quantitative methods allowed researchers to gain a more comprehensive understanding of participants' experiences during an interprofessional, service-learning trip to Guatemala. Approval for this study was received from the University of South Dakota's Institutional Review Board.

\section{Participants}

Study participants included undergraduate and graduate students from the University of South Dakota in Vermillion, South Dakota. Inclusion criteria were students must be in good standing with their respective departments, completion of an application that expressed an ability to work collaboratively with a team, having a desire to participate in international service learning, and being over the age of 18. All participants traveled to Guatemala as part of a service-learning experience through the University of South Dakota: 11 students in the spring of 2015, 15 students in the spring of 2016, 9 students in the fall of 2016, and 16 students in the spring of 2017. The students belonged to the following academic disciplines: OT $(n=21)$, PT $(n=11)$, dental hygiene $(n=5)$, PA $(n=10)$, and pre-professional undergraduate students $(n=4)$, for a total of 51 participants.

An alternate quantitative tool was used during the initial phase and therefore, participants from the spring of $2015(n=11)$ and spring of $2016(n=15)$ were not included in the quantitative portion of this study. Additionally, four students were unable to be included due to incomplete assessments. Therefore, only 21 students of the 25 that attended the fall 2016 and spring 2017 experiences were included in quantitative data analysis. The academic disciplines represented on these trips were OT ( $n=14)$, PT ( $n=$ $6)$, dental hygiene $(n=3)$, and PA $(n=2)$. All 51 students who participated in the experiences between 2015 and 2017 were included in the qualitative portion of the study. 


\section{Research Instruments}

The Transcultural Self-Efficacy Tool. The Transcultural Self-Efficacy Tool (TSET) was used to evaluate students' perception of self-efficacy in performing transcultural health care skills to diverse client populations. The TSET consists of 83 skill items that are categorized under one of three subscales: cognitive, practical, or effective (Amerson, 2010; Jeffreys, 2000). Skill items are rated on a 10-point scale ( $1=$ not confident to $10=$ totally confident) (Jeffreys, 2000). The cognitive subscale asked students to rate their knowledge on how cultural factors influence care. The practical subscale asked students to rate their confidence in interviewing individuals from different cultural backgrounds on topics such as ethnic identity and discrimination or bias. The affective subscale referred to student values, attitudes, and beliefs regarding cultural awareness, acceptance, appreciation, recognition, and advocacy (Jeffreys, 2000). Predictive validity, reliability, and internal consistency have been reported for this assessment (Jeffreys, 2000; Jeffreys \& Dogan, 2010).

Interprofessional Attitudes Scale. The Interprofessional Attitudes Scale (IPAS) was used to evaluate students' attitudes relating to the 2011 Core Competencies for Interprofessional Collaborative Practice. The IPAS is a 27 -item questionnaire that is divided into 5 subscales: teamwork, roles and responsibilities, patient-centeredness, interprofessional biases, diversity and ethics, and community centeredness. Students respond to items using a 5-point Likert scale (strongly disagree to strongly agree). Internal consistency reliability has been reported for the IPAS (Norris et al., 2015).

Nightly and Terminal Reflection. While students and faculty were in Guatemala, qualitative data was obtained through nightly written reflections and discussions guided by the following questions created by the trip leaders: (1) Heart/Feelings: What was the highlight of your day? (2) Hand/Action: Identify at least one interaction/situation today when you felt purposeful. Try to describe why you felt purposeful in that situation. (3) Head/Thoughts: What did you learn today? On the final night of the trip, a terminal reflection was also completed and was guided by the following questions: (1) How did this experience improve your critical thinking skills as future therapists or providers? (2) What was most challenging this week (and what did you learn from it)? (3) What did you learn from this experience that you plan to use in your practice once you graduate? (4) What did you learn about another discipline that you did not know before? (5) What was the biggest cultural barrier in providing care?

\section{Procedures}

Approval for this exempt study was obtained from the University of South Dakota's Institutional Review Board (IRB). Participant recruitment was conducted through informational meetings held on campus at the University of South Dakota. Meetings were advertised to students through flyers, e-mail, and faculty announcements. During the meetings, trip leaders (one OT faculty, one PT faculty, and two PA faculty) provided 
information to the students about the service-learning experience, facts about Guatemala, a tentative schedule, and estimated costs. Individuals interested in the trip contacted the trip leaders to express interest. All prospective participants were e-mailed an application form. The application for this experience included questions regarding the following: personal motivation, interactions with individuals whose beliefs differ greatly from your own, experience working with individuals who are mentally or physically disabled, program involvement, and experience with service-learning programs. All applications were reviewed and participants were chosen based on the inclusion criteria.

The study was explained to chosen participants verbally, as well as through an informed consent document. Participants were provided the consent statement but as no identifiers were collected, they were not required to sign the document. However, participants were informed that they could withdraw from the study at any time by indicating so in the first item of the pretest, posttest, or reflection writing, or by choosing not to participate in the assessments. Participants were allowed to keep the informed consent document for their records to reference in the future.

The service-learning experience investigated in this study included three components: didactic, field immersion, and debriefing. Students participate in five, one-hour educational meetings prior to immersion. This didactic component focused on cultural competence of Mayan and Latino cultures, the history of Guatemala, common conditions, wheelchair fitting and adjustments, and the healthcare system in Guatemala. Field immersion involved students providing a variety of health services to underserved populations while immersing themselves in the culture. Activities included conducting health screenings, evaluating and fitting children and adults for wheelchairs, providing fluoride applications, and completing home visits where students were able to provide families with recommendations for medical and rehabilitation needs. Cultural immersion occurred through participating in traditional meals, learning traditional dances with the local community, participating in a fútbol game with the host, visiting an elder center, and visiting historic sites. Debriefing occurred during the trip, both formally and informally. The group met each night to discuss their day, the clients, the culture, and any questions/contemplations. During the clinic each day, trip leaders worked alongside students and debriefed between clients.

Participants completed the TSET and IPAS assessments before arriving in Guatemala and after returning home. In order to ensure confidentiality, each participant created a code to use on assessments and reflections. This allowed for comparative analysis while still keeping responses anonymous. Nightly reflections and discussion were completed during each trip. The students were given 20-30 minutes to reflect on their day and write responses to the outlined prompts. After written responses were collected, the group engaged in open discussion, sharing moments of their day or 
posing questions to other group members. This occurred four nights of each trip for a total of 16 reflection sessions over the four trips. Terminal reflections were completed on the final night of the service-learning experience with a similar format as the nightly reflections, with more time given for reflection and discussion. All written responses were submitted to the trip leaders for analysis.

\section{Data Analysis}

A paired samples t-test was used to compare pretest and posttest data from the TSET and IPAS assessments. The paired samples t-test determined whether there was a significant change in students' overall mean scores after their one-week field immersion experience. Significance indicates the extent of the effect service-learning experiences had on the students' transcultural self-efficacy and attitudes toward interprofessional collaboration.

Qualitative data were analyzed using phenomenological procedures (Creswell, 2007; Moustakas, 1990). The phenomenon of interest for this study was the perceptions of student participants regarding the impact of an international, interdisciplinary service learning trip on promoting transcultural self-efficacy and attitudes towared interprofessional collaboration. Several researchers read the written reflections to get a clear sense of what participants were saying about the phenomenon and to identify the essence of each description. Essences were grouped together to form common themes, which were then articulated in a formal description. Having multiple researchers completing phenomenological analysis increases the reliability of qualitative outcomes (Leung, 2015).

\section{RESULTS}

Changes in students' scores on the TSET and IPAS were analyzed using a paired ttest; the three domains of the TSET (cognitive, practical, and affective) were analyzed separately. In the cognitive domain, representing knowledge on how cultural factors influence care, there was a statistically significant improvement from pretest to posttest $(p<.05)$. In the practical domain, representing the students' confidence in interviewing individuals from different cultures a statistically significant change occurred $(p<.05)$ as well. In the affective domain, reflecting student values, attitudes, and beliefs regarding cultural awareness, acceptance, appreciation, recognition, and advocacy, scores improved significantly $(p<.05)$. Changes in students' scores on the IPAS were not significant $(p>.05)$. See Tables 1 and 2. 
Table 1

Descriptive Statistics and t-test Results for Transcultural Self Efficacy Tool

\begin{tabular}{|c|c|c|c|c|c|c|c|c|}
\hline & \multicolumn{2}{|c|}{ Pretest } & \multicolumn{2}{|c|}{ Posttest } & & \multirow{2}{*}{$\begin{array}{l}95 \% \mathrm{Cl} \text { for Mean } \\
\text { Difference }\end{array}$} & & \\
\hline Outcome & $\bar{M}$ & SD & $M$ & SD & $\mathrm{n}$ & & $\mathrm{t}$ & $d f$ \\
\hline Cognitive & 5.28 & .56 & 7.56 & .43 & 25 & $-2.42,-2.13$ & -32.28 & 24 \\
\hline Practical & 5.17 & .45 & 7.83 & .29 & 28 & $-2.78,-2.53$ & -41.08 & 27 \\
\hline Affective & 7.64 & .62 & 8.79 & .46 & 29 & $-1.34,-.95$ & $-12.17^{*}$ & 28 \\
\hline
\end{tabular}

${ }^{*} \mathrm{p}<.05$.

Table 2

Descriptive Statistics and t-test Results for Interprofessional Attitudes Scale

\begin{tabular}{|c|c|c|c|c|c|c|c|c|}
\hline & \multicolumn{2}{|c|}{ Pretest } & \multicolumn{2}{|c|}{ Posttest } & & \multirow{2}{*}{$\begin{array}{c}95 \% \mathrm{Cl} \text { for Mean } \\
\text { Difference }\end{array}$} & & \multirow[b]{2}{*}{ df } \\
\hline Outcome & $M$ & SD & M & SD & $\mathrm{n}$ & & & \\
\hline & 5.52 & 1.88 & 5.56 & 1.95 & 45 & $-.104, .021$ & -1.333 & 44 \\
\hline
\end{tabular}

${ }^{*} \mathrm{p}<.05$.

Qualitative results indicated that engagement in international, interprofessional service learning contributed to emotional, cognitive, and skill development, as measured by positive indicators inductively derived for each category. The narrative data also explained how engagement in interprofessional, culturally diverse activities created and supported empathy and clinical competence for this group of students. For example, when students were able to provide intervention to a child with cerebral palsy, they reported the appreciation for the hands-on learning and understanding of the functional difficulties the family encountered in their impoverished environment; it provided the context and shared experience for clinical reasoning and contextual understanding. The major themes are outlined below.

\section{What was the highlight of your day?}

Uplifting Others. The idea of helping other people, and seeing firsthand the appreciation, gratitude, and happiness of the people served, was frequently identified as the highlight of the students' days. Students were moved by the outward display of affection and gratitude exhibited by the people served, as demonstrated by these two 
quotes "The smile on the girl's face when we fitted her for a wheelchair and the tears in her dad's eyes. He was so happy and grateful" and "It melted my heart when a team member told me that his mom had cried multiple times during the fitting because she was so happy."

Students felt a sense of accomplishment, pride, and internal happiness knowing that their services were benefiting the people of Guatemala. For example, "My highlight was watching our patient smile when he was tickled ... He wasn't able to do a lot physically, but his smile made my heart so happy." Another student described a similar idea, "Overall, seeing the joy, gratefulness, and happiness on the faces of those we helped and the families of those we helped. I could tell that they didn't have much simply by the looks on their faces when we helped them. It felt good to help them knowing their situations." It was also a highlight for students when they could provide a service such as described in the following quote: "Fitting a chair and stander...and seeing his face light up when he was standing level with the rest of the world." No matter the service provided, it was a highlight for students to feel as though they were assisting other people in some way, through either brightening up their day with smiles, or providing a specific service to "change lives" and uplift other people in some way.

Hands on learning in a new environment. Learning was a common highlight in students' days. Learning material in a new environment offered them novel experiences and their opportunity to interact with other professionals. For example, learning from other professionals and professions was exemplified in these two quotes: "learning about Julie's specialty" and "seeing Dr. Dean's Dental clinic set up." Other students found it very helpful to learn through hands on experiences. While in Guatemala, the students were able to interact with patients and help with their treatments. One student said, "There were many things I enjoyed today, but the greatest was getting to do a home visit with a boy who had muscular dystrophy. Owen let us feel the tone in his limbs and I really felt I learned a lot." This quote showed how the students enjoyed interacting with the patients and learning from them.

In addition to clinical learning experiences, many individuals enjoyed being immersed in a new culture and learning from it. One student said, "I enjoyed the home visits and getting to see the culture of Guatemala in a family environment. It was great to see the similarities and differences between my own family and families in Guatemala." This quote exemplified the impact and joy the students felt from getting to know a culture other than their own.

Interpersonal connections. The opportunity to meet and interact with people of a different culture was a highlight for many students, as demonstrated in the following quotes: "interacting with all the kids" and "talking to staff members of Hope Haven and learning more about their stories." Students also described the challenge of interacting 
with people who spoke a different language and yet the sense of connection and the bonds they were able to form despite the language and cultural barriers. "There was this 3-year-old named Alejandro, who was bored out of his mind, scooting a chair around the clinic. I gave him some play-dough and he became the happiest kid on earth. Regardless of language, we connected." Similar ideas were described in the following quote: "There was this little girl who wouldn't warm up to anyone. After a while, she grabbed my hand and gave me a hug. It really made me feel good and purposeful." Many students found spirituality as a way to connect with people despite the language and cultural barriers. "Going on two different home visits and simply being able to sit with the patients, listen to their stories, and discuss the hope that everyone has with Christ;" "After I helped fit a little boy to a chair, we took the photo, I shook his hand and he said, "gracias" then said something else as he pointed to me and then up toward Heaven;" and "The highlight of my day was when our group stumbled upon a church and school. We asked to go into the church, sat, and listened. Then we walked around and saw people praying. I think the fact that communication with God can be universal and meaningful is such a wonderful thing to see! It was a joy to see God working through both wheelchair fittings and prayer." Whether speaking the same language, communicating through gestures or actions or bonding through common beliefs and values, the idea of interacting with people and forming some kind of connection was an import aspect of many student's days.

Working hard to accomplish a goal. Another highlight of students' days was working hard to accomplish a goal. Many students found it rewarding to work together as a team with other professions. A student responded, "The way that our group has come together is amazing. Everyone is so willing to help each other and get the job done. We all have the same main purpose and goal." Students also felt good after working hard and putting forth a great deal of effort to help their clients and to reach a goal. One student described the highlight of her day by saying, "struggled through a wheelchair fix and new wheelchair today with a guy who had arthrogryposis. It took us a long time, but we were able to finally get him fitted." The sense of accomplishment felt by having worked hard to fix wheelchairs contributed to this individual's positive experience. Finally, after working hard and accomplishing their goals, the students often exuded pride in themselves and their abilities. As told by one individual when describing his/her favorite part of the day, "I remembered enough of my medical training to help a little girl." This quote demonstrates how one's individual capabilities and efforts can make a large impact on another person's life.

Life through a new lens. Students had the opportunity to interact with many people from diverse backgrounds and experiences. These interactions were often cited as eye opening and humbling experiences that left a positive impression on the students. This is exemplified by one individual who stated the following quote about her experience: "Just knowing what they have been through and probably what they are going through 
currently and seeing how happy they are despite all of that is very uplifting." Other times, the students' experiences rendered them feeling helpless, evidenced by one student's account, "The home visit was a great eye opening and great experience that really made my heart hurt. I wanted to help but kind of felt helpless." Despite the overwhelming feelings that resulted from this experience, it left an impact on this person and was still thought of as the best part of his or her day.

\section{Identify at least one interaction today when you felt purposeful and explain why.}

Sense of purpose from other's happiness and sense of purpose. A very common way that students felt purposeful was through feeling that in some way, whether it be large or small, they were helping to positively influence other people's lives by increasing quality of life, happiness, or just by making them feel good. It was frequently noted that students paired the idea of "purpose" with increasing other people's happiness as displayed in the following quotes: "Again, just seeing the parents reactions and how happy they were really made me feel purposeful;" and "When I was fitting a chair for a boy who seemed like he had CP and seeing the smile on his face;" and "Playing w/ a 17 year old w/ dystonia; truly felt he was happy - made me realize we are making an impact." Other students spoke of indirectly increasing happiness by making people's lives easier and helping to increase their purpose and worth as described in the following quotes: "It felt purposeful because he was able to press the buttons himself and I could tell that he felt like HE had purpose and worth," and "This patient was so stressed by the child's disability that he was overflowing with emotions throughout. To be able to make his life easier was very impactful." Similarly, another student felt purposeful by playing a role in helping to increase a child's independence and sense of purpose. "...We taught him how to use the brakes and he started to wheel around in his chair. His mom started to cry. I knew that he had little to no mobility before his wheelchair and it felt wonderful to give him that independence." This quote demonstrated how students felt an increased sense of purpose by helping other people to enhance quality of life and to find purpose.

Working together to overcome obstacles and help others. Many students felt purposeful when they had the opportunity to work together as a member of an interdisciplinary team to collaborate, problem solve and work their way through treatments, wheelchair fittings or projects while overcoming barriers. Students identified having an increased sense of purpose when they contributed ideas and utilized their skill set to help other people. Students discussed how they enjoyed working as a member of an interdisciplinary team in the following quote: "I loved working in an interdisciplinary group and I felt purpose from being able to contribute to a proper fitting for our last client - the engineering members were able to make the mechanical adjustments for an adult chair for us but wanted us to make sure the body position of the client was correct - it was great to feel needed in that team situation." While working as a member of a team, students felt purposeful when they were asked to contribute to 
the group as seen in this quote: "Other disciplines valued my opinion in asking for OT direction with patients. I felt useful to share my knowledge. Putting together a wheelchair by improving 3 headrests for a back rest - teamwork and perseverance!" They also felt a sense of purpose when they were able to ask questions of other disciplines as described in the following quote: "It was fun for me to consult with the PA and dental hygienists on why a patient has been having trouble breathing and constantly coughing the last 3 years. Patient had severe periodontal disease and caries."

In addition to feeling purposeful as a member of a multidisciplinary team, students also felt purposeful when they worked as a member of a small group to problem solve and see a project completed as described in the next quotes: "Together, we exchanged ideas and practically rebuilt a chair for a boy with muscular dystrophy," and "I felt purposeful when I helped adjust a chair from start to finish. I knew what chair to pick, and what needed to be done and I could do this without needing to seek out help," and "A classmate/team peer and I solved together how to change out front tires on an airport-type wheelchair (foldable). We utilized some assistance from the busy interpreters and "special requests" wheelchair master, but we were successful with utilizing materials available on our own." Whether it was in large or small groups, students seemed to feel a sense of purpose when they had the opportunity to contribute and work as members of a team to overcome obstacles in order to help other people and to work hard to complete complicated tasks.

Breaking down the barriers. While providing care, learning new material, and putting their skills to use, the students were able to connect with those around them. Often times, these connections led to feelings of purpose. One student described her feeling of purpose by saying, "When I made a girl who was uncomfortable and shy open up to work with us. This made me feel very connected with the child and also confirmed my desire to work with kids." This interaction with the little girl left an impact on this student. Students also felt purposeful when they were able to make a difference and exude compassion to those whom they were working with. This sense of purpose was displayed in the following quote about one individual's experience on a home visit: "not only providing a medical exam, but being able to show that you care and that they realized that." Finally, as the students were immersed in a culture different than their own, they often faced a language barrier. Breaking through this particular barrier gave some students a sense of pride and feelings of purpose, "Today I felt purposeful when we used two interpreters and were able to translate from English to Tz'utujil. It felt nice to play a role in helping translate between two completely distinct languages and unrelated languages/populations." 
Using personal gifts to provide for others. Providing a good or a service to those in Guatemala was a common source of feelings of purpose. The students felt helpful when they were able to provide the people served with something that could assist them and/or affect their life in some way. One student described this sense of purpose in his/her quote: "Helping everyone with their wheelchairs made me feel purposeful. It's really an amazing feeling knowing that you are helping people in need. Seeing how happy and excited they were really makes it worth it." Another student described his/her moment of purpose, as being a time the student gave something out of his/her own backpack to help a client. The student said, "I felt purposeful when I had ibuprofen in my backpack to give to someone who needed it. It was very cheap and meant nothing to me, but it made so much difference to her." In addition to physical goods and services that were given, another lasting service that was provided was general education. The students knew the impact that education could have on the clients' lives as it could lead to necessary lifestyle changes and ultimately benefit their future. One student was moved in this way while on a home visit. "We saw a CP patient who had a board to allow her to stand up and they didn't use it because she 'didn't like it' but educating the mom about how much it would help her was very good." Other students had feelings of helplessness in addition to their sense of purpose because they felt overwhelmed by some of the client's situations. One student perfectly described a mixed feeling in the following quote, "On the home visits I felt purposeful, but not really. I did what I could, but it was so sad because there was so much that needed to be done but I couldn't do it. I felt helpless in a way."

\section{What did you learn today?}

Life in a new world. For many students being immersed in a new culture provided an opportunity for learning. One student specified, "I learned a lot about Spanish and about the Guatemalan culture." The living conditions in Guatemala seemed shocking to many students and were frequently identified as a learning point for students as described in the following quotes: "I was incredibly taken back by the living conditions and the lack of understanding and knowledge about health" and "I learned a lot about the living conditions some Guatemalans are in. I was surprised at how poor it really was." Additionally, "...it was a huge eye opener going on the home visits and seeing how the people live." Coming from a developed country, prior to this experience some students had never been exposed to true poverty as experienced by so many of the people in Guatemala. This was evidenced by the following students statements about poverty: "The world is so, so much bigger and people are more impoverished than I ever before realized," and "today I learned that it is difficult to address the 'bigger problem' of addressing poverty and all children's needs. However, I also realized that Hope Haven and our group are taking a huge step in the right direction." The poverty also impacted the healthcare system as students learned through this experience: "I learned more about the access of acquiring basic medicines and how troubling it can be for someone living in poverty," and "We had almost none of the diagnostic tools (x-rays, MRIs, blood 
tests, UA, etc) that we have at home. These people rarely have access to medicine like most of us have in the US." By being immersed in a new culture and getting to see firsthand the healthcare system in a different country, students learned a lot and were able to see life in a "different world."

Expanding horizons. This trip helped open the eyes of many students. A great deal of them cited the poverty, culture, and living conditions as opportunities to learn and gain a new outlook on life. One student said, "I learned that there is a lot more to life than money. These people are so happy and yet have very little. It is definitely an eye opening experience." Others took this trip as an opportunity to learn about themselves. Through the hands on clinical experiences, one student learned, "to be more confident in my abilities and trust myself more." Another student learned that he/she would like to use his/her career to make big changes someday. This individual said, "Today I learned that I would definitely like to work in Latin America when I get older. I frequently question why I have been so blessed and so many others are born into poverty. This gives me motivation to get further training/more education so that I can later help those who cannot access so many resources."

Skills and knowledge to use as a professional. As the students were mainly all from healthcare disciplines, they learned many things that they will be able to use as they progress toward becoming professionals. Much of this learning came from hands on experiences when they were able to engage with the clients, "It was interesting learning more about spina bifida. It is something that I've been exposed to several times, but just hearing it explained with a patient you are working with right there makes me think I will remember it better." They also solidified many things they had learned in class through these same hands on experiences, "I learned so much by actually seeing kids with the diagnoses we have learned about in class."

In addition to being exposed to real situations, clients, and diagnoses, the students were exposed to each other and disciplines other than their own. One student said, "I learned a lot about the OT profession and their role in helping a child with MS." They also were able to learn specific skills from each other that will be useful in their own profession, "It was awesome learning from [therapist name]. He knew all these little tricks with seating and adjustments to the chair that made a huge difference." Finally, working as an interdisciplinary team helped the students see and learn firsthand the importance of teamwork in healthcare. "I learned that there is a very wide range of needs among the people here and that it takes a lot of professionals and organization to provide sustainable care." Overall, the students often identified hands on experiences and team experiences as great learning opportunities for them while on this trip. 
Therapeutic use of self. One common trend found in the learning experiences of the students was ways to effectively interact with others. As health care is a very personable profession, these learning experiences are of utmost importance. In their professions, the students will be working with people from all different backgrounds, beliefs, socioeconomic status, among other characteristics. Learning how to work with diverse populations will be important and this trip helped teach some of these lessons. As one student stated, "I learned that when you meet people where they're at culturally and personality-wise, you can make a bigger difference." Another student cited the language barrier between English and Spanish as a learning experience. This student found that you can communicate in other ways and said, "I also learned that we laugh and have fun and smile all the same language."

\section{DISCUSSION}

The phenomenon of interest in this study was how an interprofessional, international service learning experience can influence the growth of healthcare students so they become culturally sensitive professionals. The findings support this type of experience as a direct benefit to the students who participated. Specifically, the majority of students reported statistically significant improvements in transcultural self-efficacy as defined by their confidence in interviewing individuals from different cultures; their values, attitudes, and beliefs regarding cultural awareness, acceptance, appreciation, recognition, and advocacy; and their knowledge on how cultural factors influence care. Slightly different findings emerged concerning students' attitudes about interprofessional practice as measured by the IPAS. While a few students reported significantly improved attitudes, there were also students who reported significantly decreased attitudes toward interprofessional practice. It is important to note that prior to this experience, student participants were introduced to interprofessional practice through theoretical discussions and simulated scenarios only. The actual implementation of interprofessional activities may have uncovered personal biases or feelings about interprofessional interactions of which they were previously unaware. However, qualitative analysis of personal reflections uncovered that many students felt purposeful when working as part of an interprofessional team and appreciated the dialogue with other professionals when discussing patient care.

While the University's goal of developing culturally sensitive professionals was clearly supported, continued efforts must be taken to facilitate positive interprofessional interactions. Interactions between the professional faculty members were modeled, yet perhaps intentional debriefing outlining the professional interactions would have created greater impact. The overall findings of our study are corroborated by published studies examining impact on health profession students in service learning (DeBonis, 2016) interprofessional service learning (De Los Santos, McFarlin, \& Martin, 2014); and improving confidence in different cultures (Dupre, 2007; Kohlbry, 2016; Morton, 2011). 


\begin{abstract}
Limitations
Participants in our study were highly motivated students with interest in participating in a service learning experience in Guatemala and working with a culture other than their own. Having students with interest in participating in a cultural experience may have biased the outcomes positively as compared to evaluating a group of students with less inherent interest in providing culturally competent care to others. Additionally, our sample size was small and was from a single institution. A larger, more diverse sample size may have shown variations from our study results. Additionally, only written reflections were collected and discussions were not recorded and therefore not included in the qualitative analysis.
\end{abstract}

\title{
Future Research
}

Analyzing student outcomes over the ensuing years of implementation will add power to the study by increasing the sample size. Value will be added by examining why student participants have interest in participating in the service learning activity in Guatemala and/or whether they previously participated in service learning activities with other cultures. Do those students who have had prior experiences such as this differ in their responses to the survey questions? Which ones? Additionally, it would be interesting to follow students who have participated in this study out into practice. Do the students who participated in the service learning opportunity in Guatemala choose practice settings which are more culturally diverse than others in their class who did not participate? Are they more likely to practice in interprofessional settings? Moreover, evaluation of student growth in interprofessional perspectives during the service learning experience warrants further investigation with current interprofessional education assessment tools.

\section{CONCLUSION}

Overall, this study demonstrated the effectiveness of interprofessional, international service learning on cultivating culturally sensitive professionals. The personal nightly reflections articulated the deep, transformative shift that students experienced as they were exposed to the chronic needs and scarcity of basic healthcare in the communities served. Rather than cultural competence, as the students did not adopt a thorough functional knowledge of the culture, the students demonstrated cultural sensitivity as they challenged their own understanding and opened themselves to new ideas. They wrote about their awareness of others' needs in comparison to their own advantages. They were able to articulate indicators of professional growth as well, such as working hard to accomplish goals, expanding horizons, and increasing their skills and knowledge as professionals. 


\section{References}

Accreditation Council for Occupational Therapy Education. (2012). 2011 Accreditation Council for Occupational Therapy Education (ACOTE) standards. American Journal of Occupational Therapy, 66, S6-S74. https://doi.org/10.5014/ajot.2012.66S6

Accreditation Review Committee for the Physician Assistant. (2017). Accreditation Standards for Physician Assistant Education (4th Ed.). http://www.arc-pa.org/wpcontent/uploads/2016/10/Standards-4th-Ed-March-2016.pdf

Amerson, R. (2010). The impact of service-learning on cultural competence. Nursing Education Perspectives, 31(1), 18-22.

Brown, E., Munoz, J., \& Powell, J. (2011). Multicultural training in the United States: A survey of occupational therapy programs. Occupational Therapy in Health Care, 25(2-3), 178-193. https://doi.org/10.3109/07380577.2011.560240

Central Intelligence Agency. (2016). Central America and Caribbean: Guatemala. Retrieved from https://www.cia.gov/library/publications/the-worldfactbook/geos/gt.html

Commission for Historical Clarification. (1999). Guatemala memory of silence. Retrieved from https://www.aaas.org/sites/default/files/migrate/uploads/mos en.pdf

Crabtree, R.D. (2013). The intended and unintended consequences of international service-learning. Journal of Higher Education Outreach and Engagement, 17(2), 43-65.

Creswell, J. W. (2013). Qualitative inquiry and research design: Choosing among five approaches (3rd ed.). Thousand Oaks, CA: Sage.

De Los Santos, M., McFarlin, C.D., \& Martin, L. (2014). Interprofessional education and service learning: A model for the future of health professions education. Journal of Interprofessional Care, 28(4), 374-375. https://doi.org/10.3109/13561820.2014.889102

DeBonis, R. (2016) Effects of service-learning on graduate nursing students care and advocacy for the impoverished. Journal of Nursing Education, 55(1), 36-40. https://doi.org/10.3928/01484834-20151214-09

Dupre, A.M., \& Goodgold, S. (2007). Development of physical therapy student cultural competency through international community service. Journal of Cultural Diversity. 14(3), 126-134.

Humbert, T., Burket, A., Deveney, R., \& Kennedy, K. (2012). Occupational therapy students' perspectives regarding international cross-cultural experiences. Australian Occupational Therapy Journal, 59, 225-234. https://doi.org/10.1111/.1440-1630.2011.00987.x

Grusky, S. (2000). International service learning: A critical guide from an impassioned advocate. American Behavioral Scientist, 43(5), 858-867. https://doi.org/10.1177/00027640021955513

Jacoby, B. (2015). Service-learning essentials: Questions, answers and lessons learned. San Francisco, CA: John Wiley \& Sons, Inc. 
Jeffreys, M.R. (2000). Development and psychometric evaluation of the transcultural self-efficacy tool: A synthesis of findings. Journal of Transcultural Nursing, 11(2), 127-136. https://doi.org/10.1177/104365960001100207

Jeffreys, M.R., \& Dogan, E. (2010). Factor analysis of the transcultural self-efficacy tool (TSET). Journal of Nursing Measurement, 18, 120-139. https://doi.org/10.1891/1061-3749.18.2.120

Kohlbry, P.W. (2016). The impact of international service learning on nursing students' cultural competency. Journal of Nursing Scholarship, 48(3), 303-311. https://doi.org/10.1111/jnu.12209

Kumagai, A.K., \& Lypson, M. L. (2009). Beyond cultural competence: Critical consciousness, social justice, and multicultural education. Academic Medicine, 84(6), 782-787. https://doi.org/10.1097/ACM.0b013e3181a42398

Lattanzi, J., \& Pechak, C. (2011). A conceptual framework for international servicelearning course planning: Promoting a foundation for ethical practice in the physical therapy and occupational therapy professions. Journal of Allied Health, 40(2), 103-109.

Lawson, J., \& Olson, M. (2017). International service learning: Occupational therapists' perceptions of their experiences in Guatemala. The Open Journal of Occupational Therapy, 5(1), 1-12. https://doi.org/10.15453/2168-6408.1260

Leung, L. (2015). Validity, reliability, and generalizability in qualitative research. Journal of Family Medicine and Primary Care, 4(3), 324-327. https://doi.org/10.4103/2249-4863.161306

Liaison Committee on Medical Education. (2017). LCME Standards: Function and structure of a medical school. Retrieved from http://lcme.org/publications

Morton, J. (2011). Transcultural healthcare immersion: A unique interprofessional experience poised to influence collaborative practice in cultural settings. Work, 41, 303-312. https://doi.org/10.3233/WOR-2012-1297

Moustakas, C. (1990). Heuristic research: Design, methodology, and applications. Newbury Park, CA: Sage.

Norris, J., Lassche, M., Joan, C., Guo, J., Pett, M., \& Blumenthal, D. (2015). The development and validation of the Interprofessional Attitudes Scale: Assessing the Interprofessional Attitudes of Students in the health professions. Academic Medicine. https://doi.org/10.1097/ACM.0000000000000764

United States Agency of International Development (2015). Guatemala health systems assessment. Retrieved from https://www.usaid.gov/sites/default/files/documents/1862/GuatemalaHSA\%20 ENG-FULL-REPORT-FINAL-APRIL-2016.pdf

United States Census Bureau. (2012). U.S Census Bureau projections show a slower growing, older, more diverse nation a half century from now. Retrieved from http://www.census.gov/newsroom/releases/archives/population/cb12-243.html 